\title{
Le développement embryonnaire du système mélanocytaire et sa pathologie
}

Les mélanocytes de la peau constituent l'un des nombreux dérivés de la crête neurale. L'apparition de la pigmentation cutanée résulte donc d'une séquence complexe d'événements cellulaires comprenant l'individualisation des cellules de la crête neurale, la migration des précurseurs mélanocytaires depuis le primordium neural jusqu'à l'épiderme, leur multiplication cellulaire et leur différenciation en cellules productrices de mélanine. Plusieurs découvertes récentes permettent aujourd'hui de mieux comprendre certains mécanismes moléculaires et cellulaires liés au développement normal du précurseur mélanocytaire. Ces données, obtenues par des travaux de recherche fondamentale effectués essentiellement sur des embryons d'oiseau et de souris, éclairent d'un jour nouveau certaines anomalies complexes de la pigmentation cutanée chez l'homme.

\section{Catherine Dulac}

\section{ADRESSE}

C. Dulac : maître de conférences au Collège de France. Institut d'embryologie cellulairc ct moléculairc, 49 bis, avenue dc la BelleGabriclle, 94736 Nogent-sur-Marnc, France. $\mathrm{m} / \mathrm{s} n^{\circ} 4$ vol. 9 , auril 93 a couleur de la peau, de même que celle des poils des mammifères ou des plumes des oiseaux, résulte de l'activité sécrétrice de cellules très spécialisées de l'épiderme, des follicules pileux ou plumaires: les mélanocytes. Ces cellules synthétisent en effet des pigments particuliers, les mélanines, qui, transférées aux cellules épidermiques environnantes, les kératinocytes, confèrent une couleur rousse, brune ou noire sclon la nature chimique et la quantité de mélanine formée. Cependant, et contrairement aux autres cellules de l'épiderme, les cellules mélanocytaires ne sont pas produites par l'épiderme lui-même mais résultent de la migration, à un stade très précoce du développement embryonnaire, de certaines cellules de la crête neurale. La crête neurale des vertébrés est une structure embryonnaire transitoire dérivée de l'ébauche neurale primitive et qui se forme sclon un gradient rostrocaudal lors de la fermeture du tube neural (figure 1). Peu après sa formation, les cellules de la crête neurale se dissocient les unes des autres et entreprennent une migration sur des distances parfois très grandes en empruntant des voies bien définies à travers les tissus embryonnaires. Elles stoppent leur migration en des sites précis de l'embryon où elles vont achever leur processus de différenciation cellulaire pour donner naissance à des types cellulaires extrêmement variés tels que la plupart des composants du système nerveux périphérique, des cellules pigmentaires et plusicurs catégories de dérivés non neuraux ([1] et références incluses). 


\section{RÉFÉRENCES}

1. I,c Douarin NM. The Neural Crest. Cambridgc : Cambridgc University Press, 1992.

2. Lc Douarin NM. Particularités du noyau interphasique chez la caille japonaisc (Coturnix coturnix japonica). Utilisation de ces particularités comme marquage biologique. Bull Biol Fr Belg 1969 ; 103 : 435-52.

3. Le Douarin NM, Teillet MA, Couly G. Chimères cmbryonnaircs ct dévcloppement du systèmc ncrvcux. médecine/sciences 1990 ; 6 : 228-44.

4. Cohen AM, Konigsberg IR. A clonal approach to the problem of ncural crest determination. Dev Biol 1975 ; 46 : 262-80.

5. Sicber-Blum M, Cohen AM. Clonal analysis of quail neural crest cells are pluripotent and differentiate in vitro the absence of non-crest cclls. Dev Biol $1980 ; 80$ : 96-106.

6. Baroffio A, Dupin E, L,c Douarin NM. Clonc-forming ability and differentiation potential of migratory neural crest cells. Proc Natl Acad Sci USA 1988 ; 85 : 5325-9.

7. Dupin E, Baroffio A, Dulac C. Camcron-Curry P, I,c Douarin NM. Schwann-cell differentiation in clonal cultures of the ncural crest, as cvidenced by the anti-Schwann cell myelin protein monoclonal antibody. Proc Natl Acad Sci USA 1990 $87: 1119-23$.

8. Baroffio A, Dupin E, Le Douarin NM. Common precursors for ncural and mescctodermal derivatives in the cephalic neural crest. Development 1991; 112 : 301-5.

9. Teillet MA. Recherches sur le mode de migration et la différenciation des mélanoblastes cutanćs chez l'embryon d'oiscau : étude expérimentalc par la méthode des greffes hétérospécifiques entre cmbryons de caillc et de poulct. Ann Embryol Morphogen $1971 ; 4$ : 95-109.

10. Scrbedzija G, Fraser SE, BronncrFraser M. Pathways of trunk neural crest cell migration in the mousc cmbryo revealed by vital dyc analysis. Development 1990 ; $108: 605-12$.

11. Hirobe T. Control of melanocyte proliferation and differentiation in the mousc epidcrmis. Pigment Cell Res 1992 ; 5 : 1-11.

12. Mintz B. Gene control of mammalian pigmentary differentiation. I. Clonal origin of melanocytes. Proc Natl Acad Sci USA $1967 ; 58: 344-51$

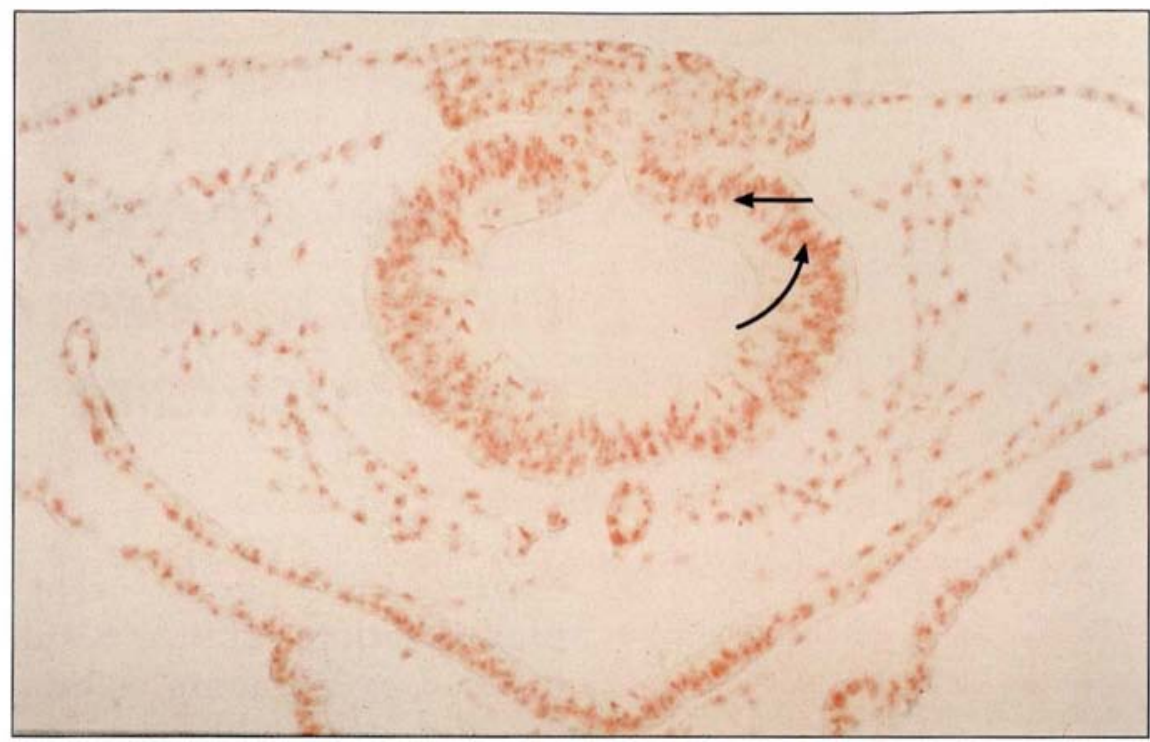

Figure 1. Formation de la crête neurale au niveau mésencéphalique d'un embryon de caille âgé d'un jour et demi (stade 7 somites). Les cellules de la crête neurale s'individualisant lors de la fermeture de la gouttière neurale forment une structure embryonnaire transitoire située entre l'ectoderme superficiel et le tube neural. Les cellules de la crête neurale vont ensuite poursuivre leur migration à travers les tissus embryonnaires jusqu'à des sites précis où elles vont achever leur processus de différenciation.

L'analyse de l'émergence des différents lignages cellulaires issus de la crête neurale a permis d'établir, pour chaque niveau de l'axe neural, l'origine embryonnaire commune des cellules pigmentaires et de certains autres dérivés de la crête, ainsi que l'existence, dans la crête en migration, de précurseurs pluripotents. Ces données permettent de mieux comprendre des maladies complexes, telles que la neurofibromatose de von Recklinghausen ou le syndrome de Waardenburg, dans lesquelles des anomalies de la pigmentation sont associées à des désordres touchant certains autres dérivés de la crête ncurale. L'identification récente de gènes dont l'altération est à l'origine de ces maladies révèle certains mécanismes moléculaires communs au développement des mélanocytes et d'autres dérivés de la crête neurale. D'autres gènes participant plus spécifiquement au contrôle de la prolifération et/ou de la différenciation des mélanoblastes ont été récemment isolés. L'altération de l'un d'entre eux au moins, le proto-oncogène $c-k i t$, a été directement corrélée à l'hypomélanose de certains patients atteints de piébaldisme* $\left(\mathrm{m} / \mathrm{s} \mathrm{n}^{\circ} 2\right.$, vol. 8, p. 185).

\section{Crête neurale et ses dérivés}

Il a été démontré dès les années 1930, par des expériences d'extirpation ou de transplantation de crêtes neurales d'embryons de vertébrés appartenant à différentes espèces, que cette structure embryonnaire est à l'origine de l'ensemble des cellules pigmentaires du corps, à l'exception de celles de la rétine, qui dérivent de la cupule optique.

La plupart des travaux récents concernant la crête neurale ont été réalisés sur l'embryon d'oiseau. En effet, l'embryon d'oiseau présente l'énorme avantage d'avoir un développement embryonnaire très proche de celui des mammifères tout en restant accessible à l'expérimentation dans l'œuf durant toute l'ontogenèse. De plus des progrès considérables ont pu être réalisés grâce à la mise au point, par Nicole Le Douarin, d'une technique de marquage cellulaire stable fondée sur une différence de structure du noyau de deux espèces d'oiscaux taxinomiquement proches : le poulet et la

\footnotetext{
* Piébaldisme : anomalie de la pigmentation des zones ventrales et des extrémités des membres.
} 
caille japonaise (Coturnix coturnix japonica) $[2,3]$. Il a été alors possible, par association de cellules des deux espèces, de construire des embryons chimères dont le développement est normal et dans lesquels les cellules d'origine caille ou poulet pourront être distinguées tout au long de l'ontogenèse, voire après l'éclosion et au cours de la vie adulte. Ainsi, le remplacement d'un segment de tube neural d'un embryon de poulet par un fragment équivalent prélevé chez un embryon de caille (figure 2) a permis non seulement d'identifier l'ensemble des structures dérivées de la crête neurale, mais également d'établir avec précision leurs niveaux d'origine le long du névraxe et les voies de migration des cellules précurseurs.

La liste des dérivés de la crête neurale ainsi établie révèle une extrême diversité de types cellulaires (Tableau I). La cartographie, grâce à l'utilisation du système caille/poulet, des niveaux d'origine de ces dérivés le long de l'axe neural montre que la crête neurale est divisée en régions qui ne fournissent qu'un nombre limité de types cellulaires. Ainsi, seule la région céphalique de la crête neurale donne naissance à la fois à des dérivés de types mésectodermique, neural et pigmentaire. La région troncale est à l'origine de la formation des ganglions nerveux sensoriels et sympathiques mais ne participe pas au développement du système nerveux entérique, qui provient de la zone vagale. Les cellules pigmentaires sont produites par la crête neurale à tous les niveaux du névraxe.

\section{Émergence de la lignée mélanocytaire}

La compréhension du processus de détermination et de différenciation des cellules pigmentaires a récemment progressé dans deux directions : (1) l'analyse des potentialités individuelles des cellules de la crête neurale, permettant d'identifier la nature des précurseurs des mélanocytes ; (2) la découverte de nouveaux facteurs synthétisés par le microenvironnement du mélanoblaste et indispensables à son développement.

La détermination, grâce à l'utilisation du système de greffes caille/poulet, des territoires présomptifs des dérivés
Tableau I

\section{DÉRIVÉS DE LA CRÊTE NEURALE}

\begin{tabular}{|c|c|}
\hline Neurones & $\begin{array}{l}\text { - Ganglions sensoriels de certains nerfs crâniaux } \\
\text { - Ganglions spinaux } \\
\text { - Ganglions des systèmes nerveux autonomes } \\
\text { sympathiques, parasympathiques et entériques }\end{array}$ \\
\hline $\begin{array}{c}\text { Cellules } \\
\text { non neuronales } \\
\text { du système } \\
\text { nerveux } \\
\text { périphérique }\end{array}$ & $\begin{array}{l}\text { - Cellules de Schwann } \\
\text { - Cellules satellites des ganglions nerveux } \\
\text { - Cellules gliales entériques }\end{array}$ \\
\hline $\begin{array}{l}\text { Cellules } \\
\text { endocrines } \\
\text { ou } \\
\text { para-endocrines }\end{array}$ & $\begin{array}{l}\text { - Cellules médullaires de la surrénale } \\
\text { - Paraganglions adrénergiques } \\
\text { - Cellules à calcitonine } \\
\text { - Cellules de type I du corps carotidien }\end{array}$ \\
\hline \multicolumn{2}{|l|}{$\begin{array}{c}\text { Cellules } \\
\text { pigmentaires }\end{array}$} \\
\hline Méninges & - Hémisphères cérébraux \\
\hline Mésectoderme & $\begin{array}{l}\text { - Squelette viscéral et facial et partie du sque- } \\
\text { lette crânial } \\
\text { - Odontoblastes } \\
\text { - Tissu conjonctif et musculaire de la paroi des } \\
\text { gros vaisseaux dérivés des arcs aortiques } \\
\text { - Tissu conjonctif des glandes pituitaires, lacry- } \\
\text { males, salivaires, thyroïde, parathyroïdes et du } \\
\text { thymus } \\
\text { - Contribution aux muscles striés de la face et } \\
\text { du cou }\end{array}$ \\
\hline
\end{tabular}

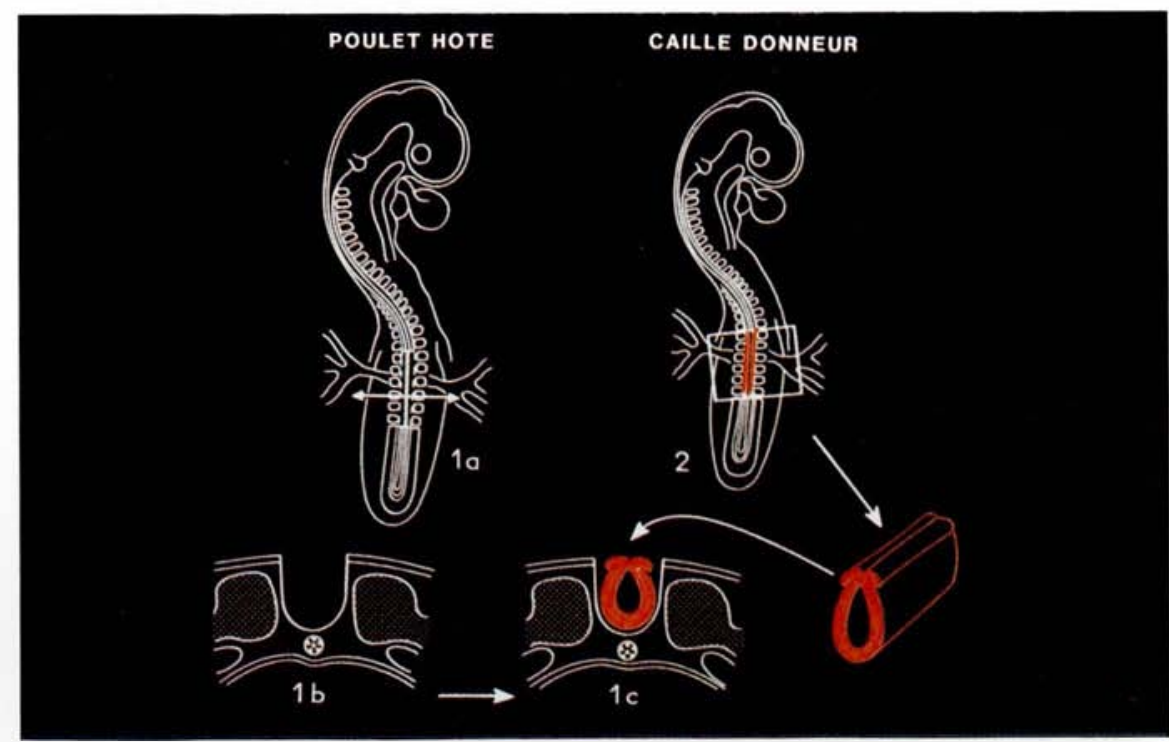

Figure 2. Réalisation de chimères de tube neural. Un fragment de tube neural est excisé chez un embryon de poulet à un niveau oú les cellules de crête neurale n'ont pas encore commencé leur migration. Un fragment équivalent est prélevé chez un embryon de caille de même stade et greffé chez le poulet. Dans l'exemple montré ici, l'âge des embryons de caille et de poulet ainsi que la position du greffon chez le donneur et l'hôte sont identiques. Ce type de greffe permet d'identifier l'ensemble des structures dérivées de la crête neu rale à ce niveau précis de l'axe neural et de suivre leurs différentes voies de migration. 


\section{RÉFÉRENCES}

13. Couly G, Lc Douarin NM. Mapping of the carly neural primordium in quailchick chimeras. II. The prosencephalic neural plate and ncural folds : implications for the genesis of cephalic human congenital abnormalitics. Dev Biol 1987 ; 120 : 198-214.

14. Ota M. Nacvus fusco-cacrulcus ophtalmo-maxillaris. Tokyo Med J 1939 ; 62 : 1243-5.

15. Enjolras O, Riche MC, Merland JJ. Facial portwinc stains and Sturge-Weber syndromc. Pediatrics $1985 ; 76: 48-51$.

16. Bolande RP. The ncurochristopathics. A unifying concept of discase arising in ncural crest maldevelopment. Hum Pathol 1974 ; $5: 409-29$.

17. Riccardi VM. Von Recklinghausen ncurofibromatosis. $N$ Engl J Med 1981; 305 : 1617-27.

18. Viskochil 1), Buchberg AM, Xu G, et al. Deletions and a translocation interrupt a clone genc at the ncurofibromatosis type I locus. Cell 1990; 62 : 187-92.

19. Wallace MR, Marchuk DA, Andersen LB, et al. Typc I ncurofibromatosis gene ; identification of a large transcript disrupted in threc FI patients. Science 1990 ; $249: 181-6$

20. Ballester R, Marchuk I), Boguski M, et al. The NFI locus encodes a protein functionaly related to mammalian GAP and ycast IRA protcins. Cell $1990 ; 63: 851-9$.

21. Xu G, Lin B, Tanaka K, Dunn D, Wood D, Gcstcland R, Whitc R, Wciss R, Tamanoï $F$. The catalytic domain of the ncurofibromatosis type I gene product stimulates Ras G'TPase and complements ira mutants of $S$. cerevisiae. Cell 1990; 63 : 835-41

22. Martin GA, Vikochil D, Bollag G, et al. The GAP-related domain of the neurofibromatosis type 1 genc product interacts with Ras p21. Cell $1990 ; 63$ : 843-9.

23. De Cluc JE, Papagcorge AG, Fletcher JA, et al. Abnormal regulation of mammalian p21 ras contributes to malignant tumor growth in von Recklinghausen (typc 1) ncurofibromatosis. Cell 1992; 69 : 265-73.

24. Basu TN, Gutman DH, Fletcher JA, et al. Aberrant regulation of ras protcins in malignant tumor cells from typc 1 ncurofibromatosis patients. Nature $1992 ; 356$ : de la crête neurale a montré que, selon leur niveau d'origine le long de l'axe neural, la destinée des cellules de crête est restreinte à un certain nombre de phénotypes. Cependant, cette régionalisation de la crête neurale en territoires présomptifs distincts ne correspond pas à une détermination rigide de ces zones vers un type particulier de différenciation. En effet, il a été montré que lorsque la position des cellules de la crête neurale est modifiée avant le début de leur migration, leur différenciation cellulaire s'effectue en accord avec le nouveau microenvironnement rencontré et non selon leur niveau d'origine. Ainsi, chaque niveau de l'axe neural peut fournir l'ensemble des dérivés de la crête neurale si ses cellules sont transplantées dans la zone adéquate de l'embryon. Cette pluripotentialité globale de la crête neurale peut résulter d'une pluripotence individuelle de chaque cellule de crête neurale ou bien de la présence d'un répertoire complet de cellules déjà déterminćes. La compréhension des mécanismes permettant l'ćmergence des différents lignages issus de la crête neurale est donc directement lice à la possibilité de connaître les potentialités de différenciation d'une cellule unique. Les premiers travaux en ce sens ont été réalisés par Cohen et Konigsberg [4] et Sieber-Blum et Cohen [5], qui ont cultivé des cellules de crête neurale troncale à de très faibles densités cellulaires. Dans ces conditions, la descendance de chaque cellule forme une colonie distincte. Leur analyse a permis d'identifier trois types de précurseurs au sein de la crête neurale : selon qu'ils engendrent des clones entièrement pigmentés, non pigmentés et mixtes. Cette étude démontra pour la première fois l'hétérogénćité des cellules de la crête neurale en ce qui concerne leur potentialité de différenciation mélanocytaire.

Une approche similaire a récemment été développée à l'Institut d'embryologie cellulaire et moléculaire qui a permis de suivre l'émergence d'un répertoire phénotypique étendu à partir de cultures de cellules uniques [6-8]. Cette technique a consisté à prélever une cellule unique à partir d'une suspension cellulaire de crête neurale mésencéphalique, à l'aide d'une micropipette et sous le contrôle du microscope. Cette cellule est ensuite déposće dans un milieu de culture complexe, sur une couche nourricière de fibroblastes de souris dont la croissance a été arrêtée par un traitement à la mitomycine. Ces conditions se sont montrées particulièrement favorables pour la prolifération cellulaire et l'émergence des principaux lignages issus de la crête neurale : diverses catégories de neurones, cellules gliales, cellules pigmentaires, dérivés mésectodermiques.

L'analyse de plus de 500 cultures clonales a montré que les cellules de crête neurale en migration possèdent des potentialités de différenciation extrêmement hétérogènes (figure 3). La présence de cellules pigmentaires a été trouvée associée à celle de cellules gliales, de neurones, de cartilage (identifiant la voie de différenciation mésectodermique), voire à la combinaison de plusieurs de ces dérivés, démontrant clairement qu'au stade de la migration des cellules de crête neurale le lignage des cellules pigmentaires n'est pas encore sćgrégé des lignages neuraux et mésectodermiques. Par ailleurs, l'extrême variété des combinaisons de phénotypes obtenues suggère qu'au cours de leur migration les cellules de la crête neurale subissent une restriction de leurs potentialités selon un mécanisme stochastique et asynchrone plutôt que sćquentiellement ordonné. Ainsi, les cellules de la crête neurale n'atteignent pas leur site d'arrêt de migration dans un état identique de détermination, et le microenvironnement va donc jouer un rôle crucial en dirigeant les précurseurs multipotentiels vers un phénotype précis et en permettant la survie et la maturation de certaines sous-populations déjà déterminées.

\section{Migration des cellules pigmentaires présomptives}

Il a pu être établi chez l'oiseau grâce au système caille/poulet [9], et récemment confirmé chez les mammifères après coloration des cellules de crête avec un colorant vital, le DiI [10], que la migration des futures cellules pigmentaires s'effectuait selon une voie bien particulière. En effet, une sous-population de cellules de crête neurale migre entre le dermomyo- 
tome et l'ectoderme selon un trajet dorso-latéral (figure 4). Après une phase de prolifération cellulaire intense dans le mésoderme sousépidermique, les mélanoblastes franchissent la lame basale sousépidermique pour coloniser l'épiderme. Ce passage du derme à l'épiderme intervient plusieurs jours après le début de la migration des précurseurs de la crête neurale [11], entre le $11^{\mathrm{e}}$ et le $12^{\mathrm{e}}$ jour de développement chez l'embryon de souris et à la $8^{\text {e }}$ semaine de vie intra-utérine chez l'homme, puis est rapidement suivi de la différenciation cellulaire des mélanoblastes en mélanocytes mûrs avec formation de dendrites et synthèse de pigments.

L'étendue de la zone cutanée pigmentéc consécutive à la greffe d'un fragment transversal de tube neural de caille chez un embryon de poulet de souche albinos à un niveau défini de l'axe neural a montré que les précurseurs des cellules pigmentaires migrent en nappe pour coloniser une zone cutanée transversale débordant largement les limites antérieure et postérieure du greffon. Une autre expérience, réalisée par agrégation de blastocystes provenant de souches de souris pigmentées et albinos [12], a permis de démontrer de façon élégante qu'un nombre limité de précurseurs suffisaient à engendrer l'ensemble de la pigmentation cutanée de la souris, chaque cellule progénitrice étant responsable d'un clone de mélanocytes disposés en une bande perpendiculaire à l'axe du corps.

La pigmentation d'un segment particulier du corps résulte donc de la différenciation d'un nombre restreint de cellules précurseurs ayant quitté l'axe neural à un niveau défini et qui partagent une origine embryonnaire commune avec des dérivés de la crête

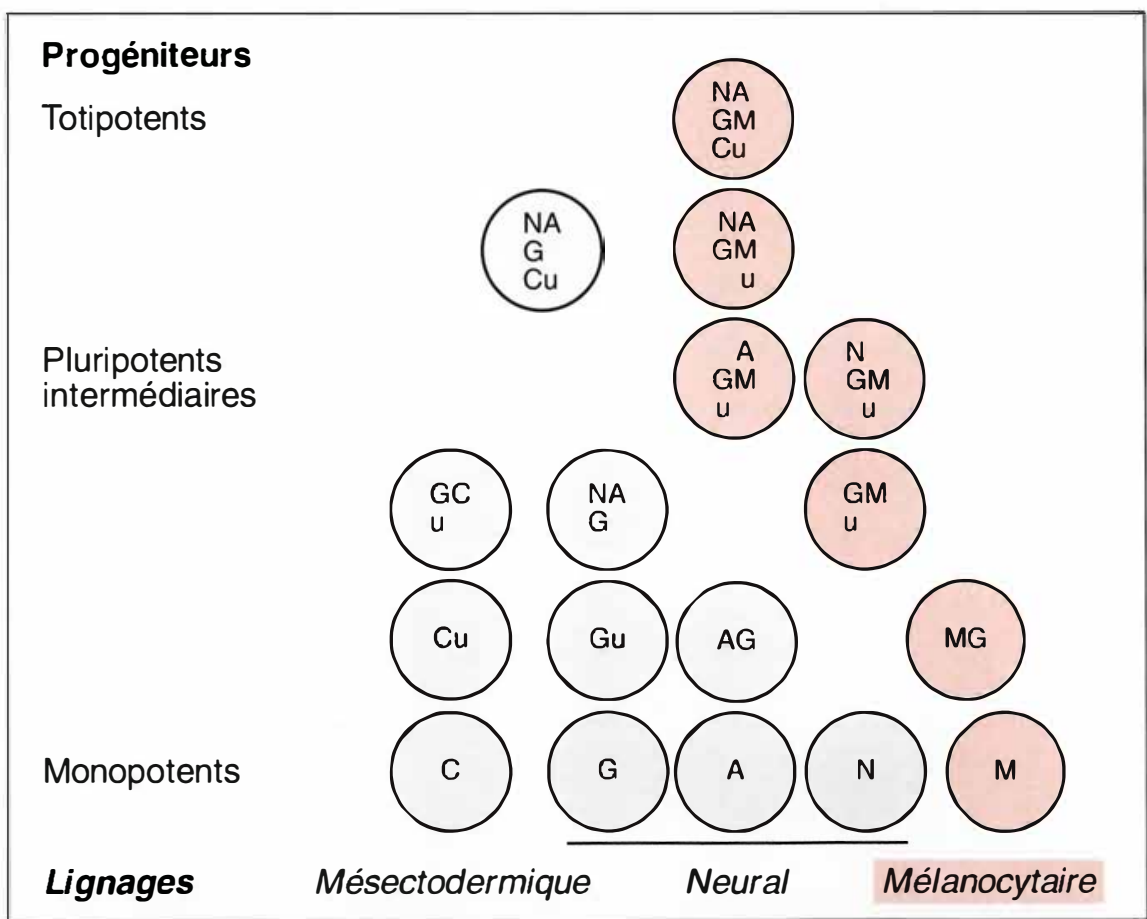

Figure 3. Schéma illustrant les différents types de progéniteurs présents dans la crête mésencéphalique en cours de migration et identifiés par l'analyse des phénotypes exprimés en cultures clonales. Chaque cercle correspond à un type de progéniteur défini et classé selon les combinaisons de types cellulaires qu'il engendre in vitro. $N$ : neurones exprimant des protéines de neurofilaments, $A$ : cellules adrénergiques identifiées par la présence de tyrosine hydroxylase, $M$ : mélanocytes identifiés par la présence de pigment, $G$ : cellules gliales, $C$ : cellules cartilagineuses ; $u$ : lignage non identifié. Les cellules de crête neurale dont la descendance contient des mélanocytes sont indiquées en rose. L'extrême diversité de phénotypes et de combinaisons de phénotypes trouvés dans les clones contenant des cellules pigmentaires démontre qu'au stade de la migration des cellules de la crête neurale le lignage mélanocytaire ne s'est pas encore individualisé des lignages neuraux et mésectodermiques.

$\mathrm{m} / \mathrm{s} \mathrm{n}^{\circ} 4 \mathrm{vol}$. 9, avril 93 neurale appartenant à d'autres lignages cellulaires. Une mutation somatique affectant un territoire précis du primordium neural est donc susceptible d'affecter la différenciation, à ce niveau du corps, de plusieurs dérivés de nature différente. Ce mécanisme a été proposé [13] pour expliquer les anomalies constatées chez des patients pour lesquels un naevus d'Ota une hypermélanose de la sclérotique et de la peau du visage innervée par les branches faciales du nerf trijumeau [14] - est associé au syndrome de Sturge-Weber, anomalie de la vascularisation du derme de la même zone du visage [15]. Dans ce cas, deux dérivés de la crête neurale sont affectés : le mésenchyme entourant les vaisseaux sanguins du visage et les cellules pigmentaires de la même région.

\section{Neurofibromatose de von Recklinghausen}

L'extrême diversité des dérivés issus de la crête neurale a souvent été corrélée à la multiplicité des catégories cellulaires impliquées dans les neurocristopathies. Le terme de neurocristopathies, proposé par Bolande en 1974 [16], regroupe un certain nombre de désordres apparemment très disparates puisqu'ils touchent des organes et des types cellulaires très variés (la peau, certains vaisseaux, des tissus nerveux, les méninges, des tissus endocrines...). Ces maladies ont cependant une pathogénie commune puisqu'elles résultent de lésions atteignant principalement des dérivés de la crête neurale.

L'exemple le plus frappant de ce type d'affections est la maladie de von Recklinghausen ou neurofibromatose de type 1 (NF1), une anomalie génétique à transmission autosomique dominante dont le locus est situé sur le chromosome 17 et dont l'incidence très élevée (une naissance sur 3500 ) en fait une des affections génétiques les plus fréquentes chez l'homme [17]. La NF1 est une maladie extrêmement polymorphe, caractérisée, dans la plupart des cas, par une pigmentation anormale de la peau (taches "café au lait"), des neurofibromes (tumeurs bénignes des nerfs périphériques), des tumeurs de l'iris dues à des amas de mélanocytes 


\section{RÉFÉRENCES}

25. I,y Y, Bollag G, Clark R, et al. Somatic mutations in the neurofibromatosis gene in human tumors. Cell 1992 ; 69 : 275-81.

26. Daston MM, Scrable H, Nordlund M, et al. The protein product of the ncurofibromatosis type 1 gene is expressed at highest abundance in ncurons, Schwann cells, and oligodendrocytes. Neuron 1992 ; 8 : 415-28

27. Ortonne JP. Picbaldism, Waardenburg's syndrome and related disorders neural crest depigmentation syndromes ? Dermatol Clin 1988 ; 6 : 205-16.

28. Epstcin DJ, Vekemans M, Gros P Splotch (Sp2h), a mutation affecting development of the mousc neural tube, shows a deletion within the paircd homcodomain of Pax-3. Cell 1991; 67: 767-74.

29. Baldwin T, Hoth C, Amos J, et al. An cxonic mutation in the HuP2 paired domain genc causcs Waardenburg's syndrome. Nature 1992 ; 355 : 637-9.

30. Tassabchji M, Read A, Newton V, et al. Waardenburg's syndrome paticnts have mutations in the human homologuc of the Pax-3 paired box genc. Nature $1992 ; 355$ : 635-6.

31. Goulding M, Chalcpakis G, Deutsh U, et al. Pax-3, a novel murin DNA binding protein expressed during carly neurogenesis. $E M B O \mathrm{~J} 1991 ; 105$ : 1135-47.

32. Moasc CE, Trasler DG. Spinal ganglia reduction in the Splotch-delayed mouse neural tubc defect mutant. Teratology $1990 ; 40$ : 67-75.

33. Moase CE, Trasler DG. Delayed ncu ral crest cell cmigration from $\mathrm{Sp}$ and $\mathrm{Spd}$ mouse ncural tube explants. Teratology 1990 ; $42: 171-82$

34. Goridis C, Brunet JF. N-CAM : structural diversity, function and regulation of expression. Semin Cell Biol 1992 ; 3 : 189-97.

35. Moase CE, Trasler DG. N-CAM alterations in Splotch neural tube defect mousc cmbryos. Development 1991; 113 : 1049-58.

36. Chabot B, Stephenson D, Chapman V et al. The proto-oncogene c-kit encoding a transmcmbranc tyrosine kinase receptor maps to the mouse W locus. Nature 1988 ; 335 : 88-9.

37. Geissler E, Ryan M, Housman D. The dominant-white spotting (W) locus of the mousc encodes the c-kit proto-oncogene. Cell $1988 ; 55: 185-92$ (nodules de Lisch), parfois associés à des gliomes optiques, un handicap intellectuel, des lésions osseuses et vasculaires, et à une incidence élevée de tumeurs malignes particulières telles que les neurofibrosarcomes, les phéochromocytomes, les astrocytomes et les rhabdomyosarcomes embryonnaires.

La prédisposition à la NF1 est la conséquence d'une altération du gène NF1 par translocation, délétion et mutation ponctuelle qui interrompent la séquence codante [18, 19]. La séquence en acides aminés correspondant à ce gène prédit une protéine hydrophile de grande taille appelée neurofibromine. Une portion de cette séquence montre une identité de $25 \%$ avec la protéine de levure IRA et une homologie plus faible avec la protéine p21 $1^{\text {ras }}$ GTPase activating protein (GAP) de mammifere [20-22]. Ces deux molécules sont des protéines inactivant le produit du gène ras en catalysant sa conversion de la forme active, liée au GTP, à la forme inactive après hydrolyse du GTP. Il a été montré que ce domaine de la neurofibromine pouvait inhiber l'activité du gène humain $H$-ras et activer in vitro l'activité GTPase de la protéine p21 ras. Des régulations anormales de Ras ont effectivement été constatćes dans des tumeurs associées ou non à la NF1 [23-25]. Le produit du gène ras jouant un rôle très important dans le contrôle de la prolifération et de la différenciation cellulaires, on peut émettre l'hypothèse que la

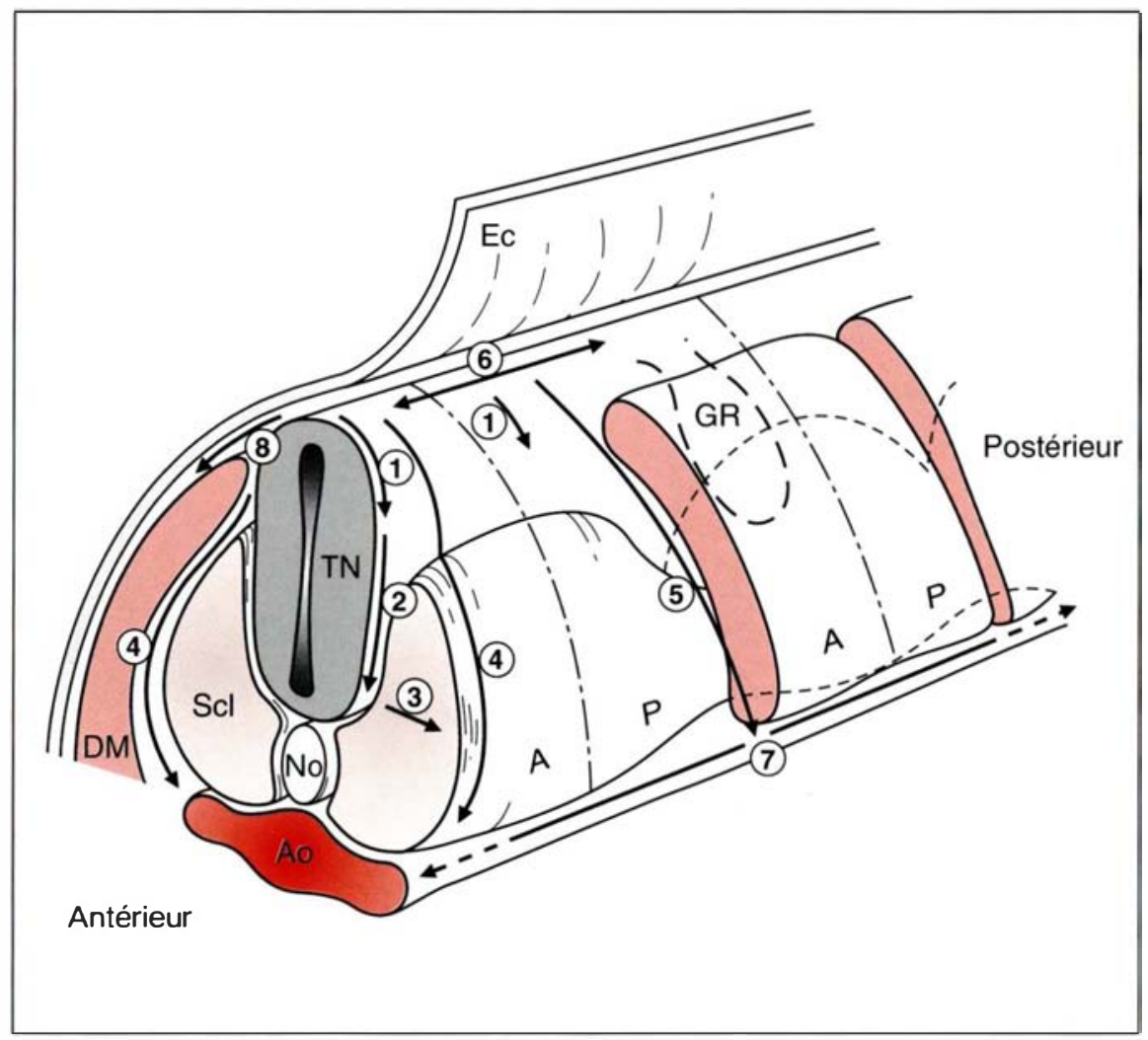

Figure 4. Représentation schématique des différentes voies de migration des cellules de la crête neurale au niveau troncal d'un embryon de 3 jours. Après avoir quitté le tube neural (TN) (1), les cellules de la crête neurale migrent selon un trajet dorso-ventral entre le tube neural et le sclérotome (Scl) (2), ou bien dans la partie antérieure (A) du somite pour participer à la formation du ganglion rachidien (GR) (3). D'autres cellules migrent entre le sclérotome et le dermomyotome (DM) (4) ou entre deux somites consécutifs (5) pour atteindre l'aorte dorsale (Ao) au niveau de laquelle se forment les chaínes sympathiques primaires. Les cellules de la crête neurale peuvent aussi suivre une voie longitudinale sous l'ectoderme (EC) (6) ou le long de l'aorte dorsale (7). Enfin, les futures cellules pigmentaires suivent une voie de migration particulière entre l'ectoderme et le dermomyotome (8). No : notochorde. 
neurofibromine normale serait impliquée dans la régulation de la multiplication et de l'état de différenciation des cellules dans lesquelles elle est exprimée $\left(\mathrm{m} / \mathrm{s} n^{\circ} 5\right.$, vol. 8, p. 471). De premières études montrent une forte expression de la neurofibromine par les cellules de Schwann et certains neurones du système nerveux périphérique, dérivés de la crête neurale, mais également par certaines cellules du système nerveux central, issues du tube neural [26]. Ces premiers résultats montrent une bonne corrélation avec les proliférations cellulaires anormales et les problèmes neurologiques constatés dans la neurofibromatose de type 1 . La maladie apparaît cependant liée à l'ensemble des dérivés neuro-ectodermiques plutôt que des seuls dérivés de la crête neurale. Des travaux plus approfondis sont encore nécessaires pour comprendre comment les mutations du gène $N F 1$ prédisposent les patients à des manifestations cliniques aussi diverses.

\section{Gène pax-3 et le syndrome de Waardenburg}

Le syndrome de Waardenburg de type 1 est une anomalie génétique humaine à transmission autosomale dominante dont le locus est situé sur le chromosome 2 [27]. Cette maladie se traduit essentiellement par des zones d'hypopigmentation de la peau et de l'iris très souvent associées à une surdité uni- ou bilatérale et pouvant s'expliquer par une anomalie du développement de la crête neurale touchant à la fois les cellules pigmentaires et les ganglions nerveux associés à l'oreille interne.

Des publications récentes montrent que le syndrome de Waardenburg, de même qu'une lignée de souris mutante, la souris Splotch, résultent d'une anomalie dans le gène pax-3 [28-30] ( $\mathrm{m} / \mathrm{s} n^{\circ} 4$, vol. 8, p. 303). Ce gène fait partie de la famille des gènes pax exprimés chez les mammifères et homologues des gènes de drosophile paired qui codent pour des facteurs de transcription contenant deux motifs se liant à l'ADN, un domaine paired et un domaine à homeobox, et impliqués dans le contrôle du développement embryonnaire. Le gène pax-3 est exprimé dans la zone dorsale du tube neural, incluant la région de l'épithélium neural d'où est originaire la crête neurale [31]. Les souris Splotch hétérozygotes ont des anomalies pigmentaires et auditives très comparables à celles observées chez les patients atteints de syndrome de Waardenburg, alors que les souris homozygotes ont des défauts touchant un nombre beaucoup plus élevé de dérivés de la crête neurale avec, en particulier, une réduction de la taille des ganglions rachidiens et des anomalies des nerfs périphériques. Ces souris présentent également des anomalies du système nerveux central telles que des anomalies de la fermeture du tube (spina bifida). Des expériences réalisées in vitro montrent que les cellules de la crête neurale de ces souris mutantes sont en nombre réduit et ont une migration retardée [32, 33]. Ces données suggèrent un rôle de pax-3 dans le contrôle très précoce du développement de la crête neurale et, plus généralement, des cellules dérivées de la zone dorsale du tube neural. Des travaux récents impliquent que les gènes de certaines molécules d'adhésion cellulaire sont des candidats intéressants comme cibles de l'action de facteurs de transcription tels que pax-3. En effet, les promoteurs de plusieurs molécules d'adhérence, telles N-CAM et la cytotactine, contiennent une séquence pouvant se lier aux homéodomaines et une expression anormale de $\mathrm{N}$ CAM a par ailleurs été reportée chez le mutant Splotch [34, 35]. L'analyse de différentes mutations touchant le gène pax-3 permettra peut-être de mieux comprendre la grande variabilité des désordres associés au syndrome de Waardenburg.

\section{Facteurs du développement des mélanoblastes}

$\mathrm{Si}$, comme nous l'avons vu précédemment, il existe de très nombreuses étapes de développement communes aux précurseurs de mélanocytes et des autres dérivés de la crête neurale, la maturation des mélanoblastes en mélanocytes implique des mécanismes spécifiques à ce lignage particulier de la crête. En particulier, les facteurs du microenvironnement cellulaire rencontré par le mélanoblaste jouent un rôle crucial pour stimuler sa prolifération et sa différenciation. L'hormone $\alpha \mathrm{MSH}$ stimule la différenciation des mélanocytes de souris in vitro par induction de l'activité tyrosinase, translocation des mélanosomes et augmentation de la dendritogenèse. Cette action se ferait, en partie du moins, par l'intermédiaire de l'AMPc. Cependant, si l' $\alpha \mathrm{MSH}$ semble être impliquée in vivo dans la variation saisonnière de la couleur du pelage de certains animaux, son action chez l'homme et son implication dans les stades embryonnaires de la mélanogenèse restent à démontrer. Le bFGF semble également intervenir dans le contrôle de la mélanogenèse en favorisant le taux de prolifération des mélanocytes de peau de souris et d'homme. Cependant, ces deux facteurs sont insuffisants pour assurer à eux seuls la mélanogenèse. En effet, leur action ne peut s'effectuer in vitro en l'absence de kératinocytes, suggérant l'existence d'autres facteurs importants produits par l'environnement des mélanocytes. Certaines de ces molécules ont pu être identifiées très récemment grâce à l'analyse de certaines souches de souris, espèce pour laquelle on connaît plus de 150 allèles représentant 60 loci affectant la couleur du pelage. En particulier, les mutations sur le locus W (white spotting) affectent, durant l'embryogenèse, le développement des mélanoblastes ainsi que, chez l'homozygote, celui des cellules souches hématopoïétiques et des cellules germinales. Les souris homozygotes sont caractérisées par une absence de pigmentation et souffrent d'anémie macrocytaire, le plus souvent létale, ainsi que de stérilité complète. L'identification du protooncogène $c$-kit comme gène du locus $\mathrm{W}$ a permis de mieux comprendre les effets pléiotropiques de cette mutation $[36,37]$. En effet, $c$-kit code pour un récepteur transmembranaire comprenant un domaine à activité tyrosine kinase et représente l'homologue cellulaire de $v$-kit. $v$-kit est l'oncogène d'un rétrovirus félin responsable de sarcomes chez le chat, et dont le caractère transformant résulte d'une délétion du domaine extracellulaire de la molécule rendant constitutive l'acti- 


\section{RÉFÉRENCES}

38. Flcischman RA, Saltman D, Stastny V, Zncimer S. Deletion of the c-kit protooncogene in the human developmental defect picbald trait. Proc Natl Acad Sci USA 1991 ; 88 : 10885-9.

39. Gicbcl I, Spritz R. Mutation of the kit (mast/stem cell growth factor receptor) protooncogenc in human picbaldism. Proc Natl Acad Sci USA 1991; 88 : 8696-9.

40. Copcland N, Gilbcrt D, Donovan 'TO, et al. Mast cell growth factor maps near the stcel locus on chromosome 10 and is delcted in a number of stecl alleles. Cell 1990 ; $63: 175-83$.

41. Zscbo K, Wypych J, Mc Nicce I, et al. Identification, purification and biological characterisation of hemapoictic stem cell factor from Buffalo rat liver-conditioned medium. Cell 1990 ; 63 : 195-201.

42. Huang E, Noika K, Bcicr D, et al. The hematopoictic growth factor KL is encoded by the SI locus and is the ligand of the ckit receptor, the gene product of the $W$ locus. Cell 1990 ; 63 : 225-33.

43. Williams DE, Eiscnman J, Baird A, et al. Identification of a ligand for the c-kit protooncogenc. Cell 1990; 63 : 167-74

44. Motro B, Van der Kooy D, Rossant J, Reith A, Berstcin A. Contiguous pattern of c-kit and stell expression : analysis of mutations at the W and SI loci. Development $1991 ; 113: 1207-21$

45. Nishikawa S, Kusakabe M, Yoshinoga $\mathrm{K}$, et al. In utero manipulation of coat color formation by a monoclonal anti-c-kit antibody : two distinct waves of c-kit dependency during melanocyte development. vité tyrosine kinase. Ainsi, c-kit pourrait être impliqué dans le contrôle de la prolifération et/ou de la différenciation cellulaire normale des cellules dans lesquelles il est exprimć. Le phénotype de la souris mutante hétérozygote étant très semblable à celui de patients atteints de piébaldisme, une anomalie de la pigmentation caractérisée par une dépigmentation des zones ventrales et des extrémités des membres, l'ADN de plusieurs patients a été analysé. Dans un petit nombre de cas, le gène $c$-kit apparaît effectivement anormal [38, 39] $(\mathrm{m} / \mathrm{s}$ $n^{\circ} 2$, vol. 8, p. 185).

Les mutations sur un autre locus, le locus Steel, causent des anomalies dans les trois mêmes lignées - mélanocytaire, hématopoïétique et germinale - que les mutations du locus W. Il existe cependant une différence fondamentale entre les deux types de mutations : les mutations du locus $\mathrm{W}$ affectent directement les cellules souches mélanocytaires, hématopoïctiques et germinales et le déficit dans l'un de ces lignages peut être corrigé par un apport de cellules souches d'une souris normale. Au contraire, la greffe de cellules souches d'une souris normale chez unc souris mutée sur le locus Steel ne corrige pas la mutation, alors que les cellules souches des souris mutées transplantées dans une souris normale se développent normalement. Ainsi, les mutations du locus Steel atteignent le microenvironnement des cellules souches et non les cellules souches ellesmêmes $\left(\mathrm{m} / \mathrm{s} n^{\circ} 10\right.$, vol. 6, p. 1016). Il a été montré que le produit du gène Steel appelé SCF (stem cell growth factor), MGF (mast cell growth factor), KL (kit-ligand) ou Sf (Steel factor) est en fait le ligand de la molécule codée par $c$ kit. Le gène Steel pourrait donc représenter un autre candidat pour expliquer les cas de piébaldisme où le gène $c$-kit est normal [40-43].

L'identification du couple de gènes $c$ kit/Steel ouvre des perspectives extrêmement intéressantes pour mieux comprendre la biologie des trois types de cellules souches associées. Ainsi, les gènes Steel et c-kit sont exprimés sur les lieux de prolifération et de migration des mélanoblastes. De plus, l'injection in utero d'anticorps bloquant la fonction du récepteur codé par le gène $c$-kit au stade de la proli- fération intradermique des mélanoblastes et de leur pénćtration dans l'épiderme engendre des souris nouveau-nćes totalement dépigmentées alors que des injections à des stades antérieurs ou ultérieurs n'altèrent la coloration du pelage que de façon mineure. Cela suggère un rôle crucial du couple de gènes $c$-kit/Steel lors d'une étape très particulière du développement des mélanoblastes. De plus, l'expression des transcrits de ces deux gènes dans des zones inattendues, telles que certaines zones très particulières du cerveau, font soupçonner un rôle beaucoup plus large au cours du développement et éventucllement des aspects pathologiques passés jusqu'à présent inaperçus $[44,45]$

\section{Remerciements}

L'auteur remercie Madame le professeur Nicole Le Douarin et Anne Baroffio pour leur lecture critique de ce manuscrit.

\section{TIRÉS A PART}

C. Dulac.

\section{Summary}

The embryonic development of melanocytes and its pathology

Melanocytes of the skin do not originate from the epidermis but from a transient embryonic structure called the neural crest, which derives from the neuroectoderm. The skin pigmentation involves a set of complex developmental mecanisms occurring outside as well as inside the embryonic skin, such as the emergence of the melanocyte cell lineage among neural crest cells, the migration of melanocyte precursors from the neural primordium to the epidermis, cell proliferation and final maturation of melanoblasts into melanin producing cells. Recently published data on avian and rodent neural crest cell and melanocyte development lead to a better understanding of normal melanogenesis and of complex abnormalities of human skin pigmentation. 